\title{
C+C Fusion Cross Sections Measurements for Nuclear Astrophysics
}

\author{
S. Almaraz-Calderon ${ }^{1, a}$, P. F. F. Carnelli ${ }^{1,2}$, K. E. Rehm ${ }^{1}$, M. Albers ${ }^{1}$, M. Alcorta ${ }^{1, b}$, P. F. Bertone ${ }^{1, c}$, B. Digiovine ${ }^{1}$, \\ H. Esbensen ${ }^{1}$, J. O. Fernandez Niello ${ }^{2}$, D. Henderson ${ }^{1}$, C. L. Jiang ${ }^{1}$, J. Lai ${ }^{3}$, S. T. Marley ${ }^{1}$, , O. Nusair ${ }^{1}$, T. Palchan- \\ Hazan $^{1}$, R. C. Pardo ${ }^{1}$, M. Paul ${ }^{4}$, and C. Ugalde ${ }^{1}$ \\ ${ }^{1}$ Physics Division, Argonne National Laboratory. Argonne, IL 60439, USA. \\ ${ }^{2}$ Laboratorio Tandar, Comisión Nacional de Energía Atómica, B1650KNA San Martín, Buenos Aires, Argentina. \\ ${ }^{3}$ Department of Physics and Astronomy, Louisiana State University, Baton Rouge, Louisiana, 70803, USA. \\ ${ }^{4}$ Racah Institute of Physics, Hebrew University, Jerusalem 91904, Israel.
}

\begin{abstract}
Total fusion cross section of carbon isotopes were obtained using the newly developed MUSIC detector. MUSIC is a highly efficient, active target-detector system designed to measure fusion excitation functions with radioactive beams. The present measurements are relevant for understanding x-ray superbursts. The results of the first MUSIC campaign as well as the astrophysical implications are presented in this work.
\end{abstract}

\section{Introduction}

Fusion reactions among carbon isotopes play an important role in studies of nuclear structure and nuclear astrophysics. The study of these reactions can help to elucidate problems from fundamental nuclear structure to astrophysical explosions. In nuclear structure for example, there are reported oscillations in the ${ }^{12} \mathrm{C}+{ }^{12} \mathrm{C}$ fusion cross section data which have not been observed in the neighboring systems [1]. Is therefore important to re-measure some of the data and extract the behavior for the ${ }^{12} \mathrm{C}+{ }^{12} \mathrm{C}$ fusion excitation function as well as for the neighboring carbon systems which have not yet been studied. In nuclear astrophysics, recent calculations suggest that fusion of neutron rich isotopes of $\mathrm{C}, \mathrm{O}, \mathrm{Ne}$ and $\mathrm{Mg}$ may play a role in triggering the recently observed explosive stellar events called $\mathrm{x}$-ray superbursts [2-4]. Although most of the nuclei involved in the calculations are outside of our current experimental capabilities, it is critical to test those calculations and compare them with experimental data when possible. We have performed measurements of the total fusion cross section of carbon isotopes $\left({ }^{10,12,13,14,15} \mathrm{C}+{ }^{12} \mathrm{C}\right)$ using the newly developed MUSIC detector. MUSIC is a highly efficient, active target-detector system designed to measure fusion excitation functions with radioactive beams. The results of the first MUSIC campaign as well as the astrophysical implications are presented in this work.

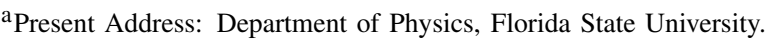
Tallahassee, FL 32306, USA. e-mail: salmaraz@physics.fsu.edu

${ }^{b}$ Pressent Address: TRIUMF, Vancouver, British Columbia VGT 2A3, Canada.

${ }^{\mathrm{c}}$ Pressent Address: Marshall Space Flight Center, Huntsville, Alabama 35812, USA

${ }^{\mathrm{d}}$ Pressent Address: Department of Physics, University of Notre Dame, Notre Dame, IN 46556, USA.
}

\section{Carbon Burning in Stars}

The ${ }^{12} \mathrm{C}+{ }^{12} \mathrm{C}$ fusion reaction is an important energy source during the late stages of stellar evolution as well as in stellar explosions such as type Ia supernovae and $\mathrm{x}$-ray bursts [5]. X-ray bursts are rapid increases in X-ray luminosity which occur in the envelopes of accreting neutron stars. The hydrogen/helium rich material being accreted on the surface of a neutron star from a companion star, will increase its temperature and density until is high enough to trigger a thermonuclear explosion. The hydrogen and helium fuel will be captured and converted into exotic proton rich nuclei via the rapid proton capture process [6]. The energy released by a typical x-ray burst is of the order of $10^{39-40}$ ergs and last about 10-100 sec. Superbursts are 1000 times more energetic and 1000 times longer in duration than typical x-ray bursts but with similar spectral evolution, which suggests that they are thermonuclear flashes involving carbon burning from the ashes of normal x-ray bursts at much larger depths than the typical ones [7]. Recent calculations show that reactions among neutron-rich carbon, oxygen and neon nuclei may be responsible for rising the temperature in the 'ocean' of the neutron star enough to strongly affect the highly temperature-dependent carbon burning [2-4]. Fusion reactions have been a very active and successful field during the last decades, mainly involving stable beams [8]. Measurements of fusion cross sections with radioactive beams are hampered to a large extent by the low beam intensities of these beams at the existing facilities.

\section{The MUSIC Detector}

Using an alternative approach, we have developed the MUSIC detector, a Multi-Sampling Ionization Chamber 
operated in active target and detector mode, designed to efficiently measure several points of a fusion excitation function with a single energy radioactive beam. The MUSIC is a medium-sized gas-filled detector which measures energy losses of the beam along a particle track. It is housed inside a $30 \times 20 \times 10 \mathrm{~cm}^{3}$ aluminum box which, during the present experiments, was filled with $\mathrm{CH}_{4}$ gas at 200 mbar, providing the ${ }^{12} \mathrm{C}$ targets. It is important to point out that other suitable counting gasses (e.g. He, $\mathrm{Ne}, \mathrm{Ar}, \ldots$ ) can be used as well.

The schematic of MUSIC is shown in Fig 1. The beam enters the detector through a $1.45 \mathrm{mg} / \mathrm{cm}^{2} \mathrm{Ti}$ window. When the beam travels through the detector it loses energy by interacting with the ionization gas which is used, at the same time, as target for the reaction as well as detection medium. Fusion events occur at different energies depending on the position inside the detector creating evaporation residues (ER). The stopping power of an ER is considerable higher than the energy loss from a beam particle leading to a sudden increase of the detected energy loss $\Delta \mathrm{E}$ at the position where a fusion reaction occurred followed by a few high $\Delta \mathrm{E}$ signals and finally its disappearance since the ER has a shorter range than the beam and stops within the detector.

The anode of the detector provides the multi-sampling capabilities. It is segmented into 18 strips as shown in the lower part of Fig. 1. The signals from the first and last strips ( $\triangle \mathrm{E} 0$ and $\Delta \mathrm{E} 17)$ are used as veto signals to eliminate reactions occurring at the entrance and exit windows. Strips 1 to 16 are called 'active strips' and are subdivided into left (L) and right (R) as shown in Fig. 1. The left and right division of the active strips is used as a "multiplicity filter' to e.g. discriminate against elastic scattering events which may be misinterpreted as fusion. While beamlike and fusion events have multiplicity one, meaning signals in either the right or the left of the anode strips, elastic scattering events produce multiplicity two events, meaning signals occur on both sides at the same time. This information is then used to reject such scattering events in an event-by-event basis.

Most of the time the beam particles pass through the MUSIC detector with no other interaction. Their energy loss signals in the gas can be seen by the black traces in Fig. 2, where the trace of a ${ }^{12} \mathrm{C}$ beam can be identified by its energy loss signals in each strip. Some typical fusion events are shown by the red traces in Fig. 2, with fusion occurring in strip 2. These events are characterized by beam like traces in the first two strips followed by a sudden jump in energy loss at strip 2 as a result of the creation of an ER. The next few strips also show higher beam energy loss signals corresponding to the ER before the signal goes to zero as the ER gets stopped due to its higher stopping power. The response of the MUSIC detector was simulated with the Monte Carlo code FLUKA [9] with very good agreement between the simulations and the experimental data. Further details on the MUSIC detector can be found on Refs. [10,11].
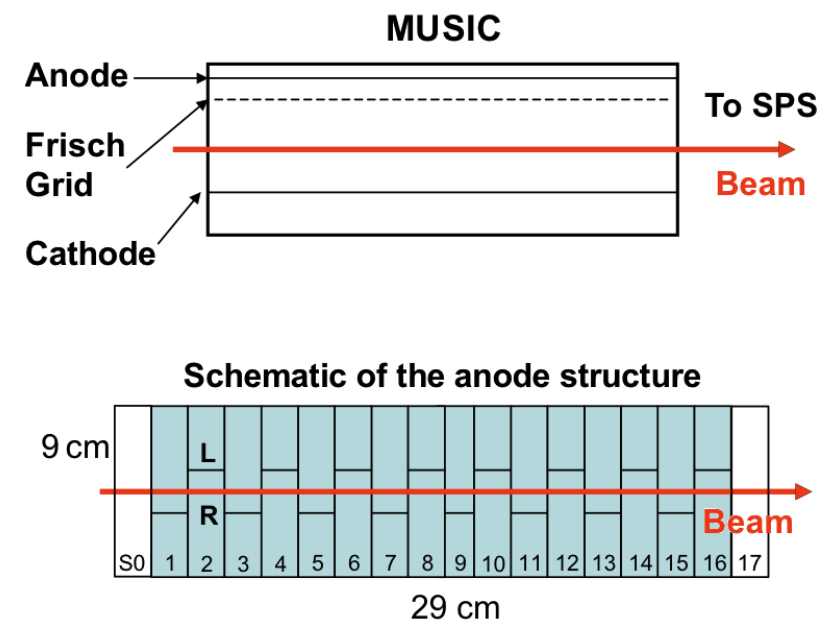

Figure 1. Schematic view of the MUSIC detector. The upper part shows the schematics of the full detector. The lower part shows the schematics of the anode structure that provides the multi-sampling capabilities. (Taken from Ref. [10].)

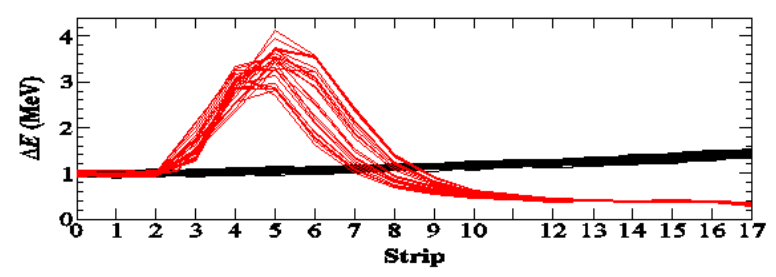

Figure 2. Traces of events inside the MUSIC detector. Beam like events are shown by the solid black lines. The beam passes through the detector leaving an easy to recognize energy loss trace. Fusion events occurring in strip 2 are also shown in the figure by the red solid lines. The main characteristics of fusion events are the jump in the energy loss signal followed by a few high energy loss signals. The trace then goes to zero as the evaporation residues are stopped due to their higher stopping power. (Taken from Ref. [10].)

\section{First Experimental Campaign with MUSIC}

The experiments were carried out at the ATLAS facility at Argonne National Laboratory. Beams of ${ }^{10} \mathrm{C},{ }^{12} \mathrm{C},{ }^{13} \mathrm{C},{ }^{14} \mathrm{C}$ and ${ }^{15} \mathrm{C}$ were used for this set of experiments. The energy of the carbon beams was between 40 to $50 \mathrm{MeV}$. Stable ${ }^{12} \mathrm{C}$ and ${ }^{13} \mathrm{C}$ beams were used as proof of principle to remeasure the ${ }^{12} \mathrm{C}+{ }^{12} \mathrm{C}$ and ${ }^{13} \mathrm{C}+{ }^{12} \mathrm{C}$ fusion excitation functions and compare them with existing data in the corresponding energy regions. For the production of ${ }^{14} \mathrm{C}$ beam, a ${ }^{14} \mathrm{C}$ radioactive sample $\left(\mathrm{t}_{1 / 2}=5730 \mathrm{y}\right)$ was mounted in the negative-ion sputter source of the tandem accelerator at Argonne National Laboratory. The short-lived isotope beams of ${ }^{10} \mathrm{C}\left(\mathrm{t}_{1 / 2}=19.3 \mathrm{~s}\right)$ and ${ }^{15} \mathrm{C}\left(\mathrm{t}_{1 / 2}=2.45 \mathrm{~s}\right)$ were generated at the ATLAS accelerator via the in-flight technique using primary beams of ${ }^{10} \mathrm{~B}$ and ${ }^{14} \mathrm{C}$ and the inverse reactions ${ }^{1} \mathrm{H}\left({ }^{10} \mathrm{~B},{ }^{10} \mathrm{C}\right) \mathrm{n}$ and ${ }^{2} \mathrm{H}\left({ }^{14} \mathrm{C},{ }^{15} \mathrm{C}\right) \mathrm{p}$ respec- 


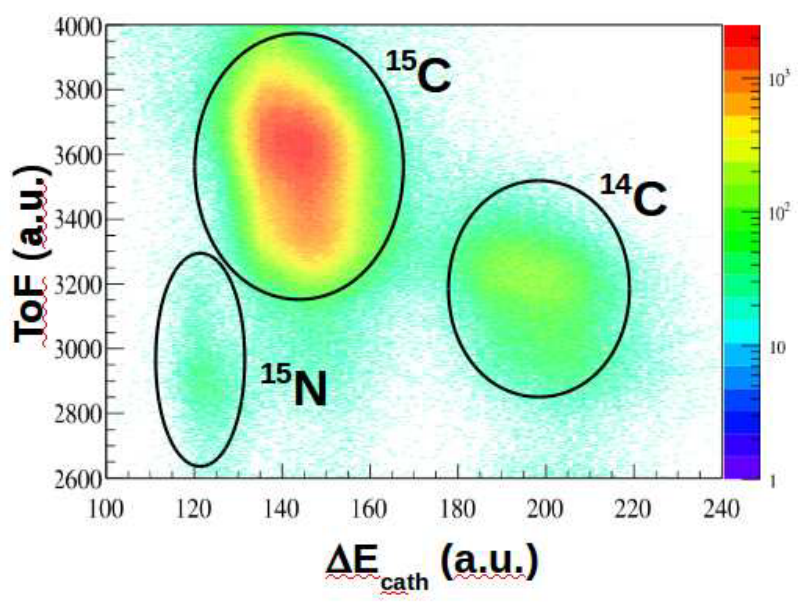

Figure 4. Energy loss in the cathode versus ToF spectrum where it is shown that the secondary ${ }^{15} \mathrm{C}$ beam can be separated from the contaminants $\left({ }^{15} \mathrm{~N}\right.$ and $\left.{ }^{14} \mathrm{C}\right)$ in the MUSIC detector.

tively. The beams were then transported to the MUSIC detector through a $26^{\circ}$ bending magnet and a radio-frequency sweeper system which eliminated most of the scattered primary beam particles [12]. The results of the experiments carried out in the first MUSIC campaign are shown in Fig. 3. The fusion excitation functions of the systems ${ }^{12} \mathrm{C}+{ }^{12} \mathrm{C},{ }^{13} \mathrm{C}+{ }^{12} \mathrm{C}$ and ${ }^{14} \mathrm{C}+{ }^{12} \mathrm{C}$ are shown in comparison with previous data. It can be seen that MUSIC data are in very good agreement with previously reported data giving us confidence in our technique. The fusion excitation functions with radioactive ${ }^{10} \mathrm{C}$ and ${ }^{15} \mathrm{C}$ beams on ${ }^{12} \mathrm{C}$ are reported for the first time.

At present the main limitation of the MUSIC detector is the low count rate it can tolerate in order to avoid pileup effects leading to the incorrect identification of fusion events. At the ATLAS facility with its bunched beam, this is achieved by requiring one particle per bunch and separating each bunch in intervals of around $5 \mu \mathrm{s}$, which is the typical drift time of electrons in the ion chamber. A radio-frequency sweeper is used with the bunching system to assure these conditions. For radioactive beam experiments where the beam intensities are low, this limitation does not hinder the performance of MUSIC. Since radioactive beams typically have contaminants due to the production method it was important to evaluate the performance of MUSIC under 'real' conditions. For example of a ${ }^{15} \mathrm{C}$ beam which was produced using the ${ }^{2} \mathrm{H}\left({ }^{14} \mathrm{C},{ }^{15} \mathrm{C}\right) \mathrm{p}$ reaction the main contaminants are ${ }^{14} \mathrm{C}$ from the primary beam, as well as ${ }^{15} \mathrm{~N}$ from the ${ }^{2} \mathrm{H}\left({ }^{14} \mathrm{C},{ }^{15} \mathrm{~N}\right)$ n reaction. The time of arrival of the beam bunches at MUSIC along with the energy loss in either the first control strip or the cathode allows for a clean separation of the particles of interest as shown in Fig. 4.

Some of the main advantages of the MUSIC detector are:

1) The efficient measurement of an excitation function using a beam with a single energy. The typically low rates of radioactive beams make them difficult to tune and therefore changing energies could lead to considerable down times during the experiment. With MUSIC one measures a range of an excitation function with a single beam energy. Moreover, it is possible to adjust the pressure inside the detector to change the energy range of the measurement which is much easier than to re-tune the radioactive beam.

2) The flexibility to use different targets. The results reported in this work were obtained with $\mathrm{CH}_{4}$ as counting gas and target. Other counting gases are well suited to be used with MUSIC, in fact, experimental campaigns with ${ }^{4} \mathrm{He},{ }^{20} \mathrm{Ne}$ and ${ }^{22} \mathrm{Ne}$ are currently underway.

3) The measurements are self-normalizing. Since the beam is being sampled all the time and is the same for all the strips, we have an absolute normalization without the need of a separate monitor detector which can introduce additional uncertainties to the excitation function.

We are presently working to eliminate the count rate limitations experienced for experiments involving stable beams.

\section{Astrophysical Implications}

The fusion cross section for the $\mathrm{C}+\mathrm{C}$ systems measured in the present work converted into astrophysical S-factors and plotted as a function of the energy are shown by the solid points in Fig. 5. The current experimental data are compared with calculations by Yakovlev et al.[3] (solid lines) and with other available experimental data in the energy range shown in the figure $[1,14]$ (open red points). There is a remarkable agreement over the full energy range between theory and experiments. Small deviations can be seen at high energies $\left(\mathrm{E}_{c m}>14 \mathrm{MeV}\right)$. Although fusion reactions predicted to occur in the interiors of neutron stars like ${ }^{24} \mathrm{C}+{ }^{24} \mathrm{C}$ cannot be measured in the near future, comparisons like the one presented here are very encouraging and allows to calibrate the calculations for cases that cannot yet be studied in the laboratory.

\section{Summary}

We have developed a highly efficient active target-detector system for measuring fusion excitation functions with radioactive beams. For the present experiments, the MUSIC detector was filled with $\mathrm{CH}_{4}$ gas which was used as counting gas and provided the ${ }^{12} \mathrm{C}$ targets. Test experiments with stable ${ }^{12} \mathrm{C}$ and ${ }^{13} \mathrm{C}$ beams were performed and the results are in very good agreement with data from the literature. We measured fusion excitation functions of carbon systems with radioactive beams, ${ }^{14} \mathrm{C}+{ }^{12} \mathrm{C}$ and, for the first time, ${ }^{10} \mathrm{C}+{ }^{12} \mathrm{C}$ and ${ }^{15} \mathrm{C}+{ }^{12} \mathrm{C}$. The high efficiency of the MUSIC detector will allow for future measurements with other neutron-rich beams. By using different counting gasses, like $\mathrm{He}, \mathrm{Ne}$, Ar; fusion excitation functions of several systems can be studied. The present measurements are of interest in nuclear astrophysics where, in the crust of accreting neutron stars, neutron-rich isotopes are thought to be responsible for opening the path to $\mathrm{x}$-ray superbursts. 

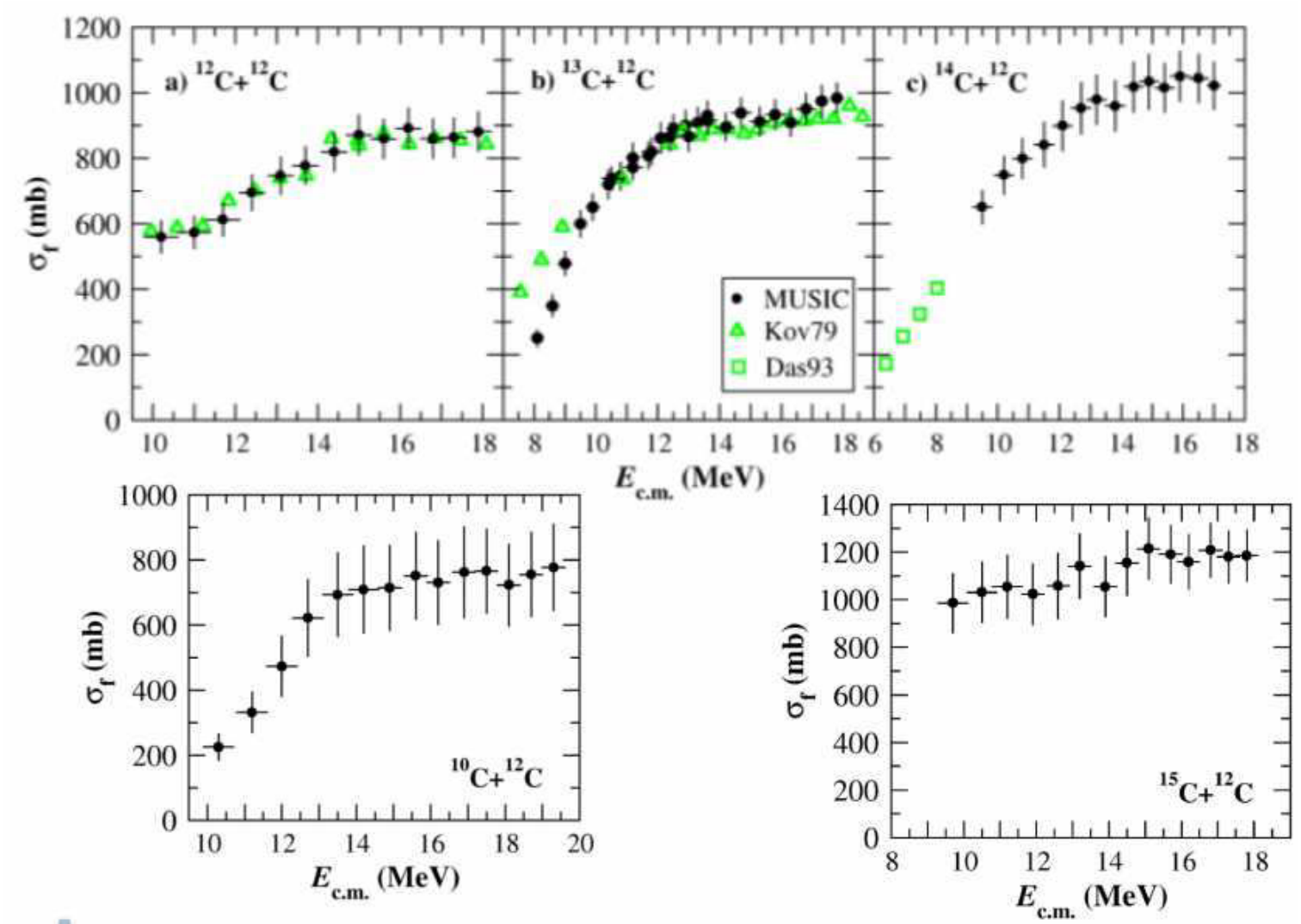

Figure 3. Experimental cross sections measured with the MUSIC detector. (Taken from Ref. [13].)

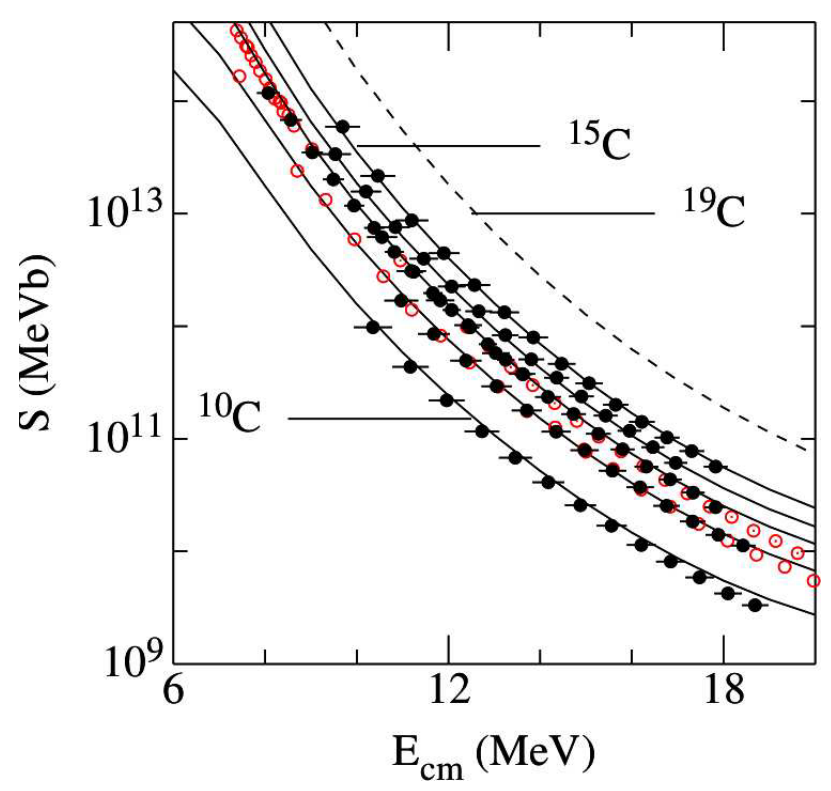

Figure 5. Solid points: $\mathrm{C}+\mathrm{C}$ fusion data measured in the present work using the MUSIC detector and converted to S-factors. Open red points: Experimental data converted to S-factor taken from Refs. [1, 14]. Solid lines: Theoretical S-factor taken from the calculations by Yakovlev et al. [3]. (Taken from Ref. [11].)

In the energy region accessible with existing radioactive beams, a good agreement between experiments and calcu- lations is observed. This gives confidence and allows to calibrate the calculations for cases that are out of reach of our current and foreseeable experimental facilities.

This work was supported by the US Department of Energy, Office of Nuclear Physics under contract No. DEAC02-06CH11357.

\section{References}

[1] D. G. Kovar et al., Phys. Rev. C 20, 1305 (1979).

[2] C. J. Horowitz, H. Dussan, and D. K. Berry, Phys. Rev. C 77, 045807 (2008).

[3] D. G. Yakovlev, M. Beard, L. R. Gasques, and M. Wiescher, Phys. Rev. C 82, 044609, (2010); M. Beard, A. V. Afanasjev, L. C. Chamon, L. R. Gasques, M. Wiescher, and D. G. Yakovlev, At. Data Nucl. Data Tables 96, 541 (2010).

[4] A. S. Umar, V. E. Oberacker, and C. J. Horowitz, Phys. Rev. C 85, 055801 (2012).

[5] G. Wallerstein et al., Rev. Mod. Phys. 69, 995 (1997).

[6] H. Schatz and K. E. Rehm, Nucl. Phys. A 777, 601 (2006).

[7] A. Cumming and L. Bildsten, Astrophys. J. 559, L127 (2001). 
[8] B. B. Back, H. Esbensen, C. L. Jiang, and K. E. Rehm, Rev. Mod. Phys. 86, 317 (2014) and references therein.

[9] G. Battistoni et al., AIP Conf. Proc. 896, 31-49 (2007).

[10] P. F. F. Carnelli et al., Nucl. Instrum. Methods. Phys. Res., Sect. A, (to be published).
[11] P. F. F. Carnelli et al., Phys. Rev. Lett. 112, 192701 (2014).

[12] B. Harss et al., Rev. Sci. Instr. 71, 380-387 (2000).

[13] P. F. F. Carnelli. PhD Thesis. Universidad Nacional De General San Martin. Comision Nacional de Energia Atomica. Buenos Aires, Argentina (2014).

[14] R. A. Dayras, R. G. Stokstad, Z. E. Switkowski, and R. M. Wieland, Nucl. Phys. A 265, 153 (1976). 